\title{
Dynamics of three simultaneously stored beams in a storage ring
}

\author{
M. Attal, ${ }^{1, *}$ P. Brunelle, ${ }^{2}$ A. Loulergue, ${ }^{2}$ A. Nadji, ${ }^{2,1}$ L. Nadolski, ${ }^{2}$ and M.-A. Tordeux ${ }^{2}$ \\ ${ }^{1}$ SESAME, P.O. Box 7, Allan 19252, Jordan \\ ${ }^{2}$ Synchrotron SOLEIL, Saint Aubin, BP 34, 91192 Gif-sur-Yvette, France
}

(Received 20 November 2011; revised manuscript received 27 December 2012; published 6 May 2013)

In this study, the observation of three simultaneously rotating beams in the SOLEIL storage ring is reported. This event occurred in November 2007 while operating in a low momentum compaction factor mode. The dynamics of the three beams is simulated using the longitudinal equations of motion and the longitudinal phase-space Hamiltonian which are extended to include higher-order terms of momentum compaction factor up to the third order. The effect of the transverse oscillation amplitude of the particles is also included in this work. It is shown that this term, which is experimentally very difficult to compensate for very low momentum compaction factor optics, can strongly affect the longitudinal beam dynamics. Finally, an extended formula of the variation of synchrotron frequency with respect to the relative variation of the rf frequency is derived and is used to deduce the higher-order terms of the momentum compaction factor from experimental data.

DOI: 10.1103/PhysRevSTAB.16.054001

PACS numbers: 29.27.Bd, 29.20.dk

\section{INTRODUCTION}

A series of experiments has been conducted since 2007 at the SOLEIL synchrotron light source [1] to obtain picosecond electron bunch length conditions to produce infrared coherent synchrotron radiation in the Terahertz region and, at the same time, to allow time-resolved $\mathrm{x}$-ray experiments $[2,3]$.

In the low bunch current regime, disregarding collective beam effects, the bunch length scales with the square root of the momentum compaction factor $\alpha$. A new lattice optics for the SOLEIL storage ring has then been optimized to reach lower momentum compaction factors (low $\alpha$ ). In this optics, the first and second-order momentum compaction terms $\alpha_{1}$ and $\alpha_{2}$ are reduced using quadrupole and sextupole magnets, respectively, while the horizontal emittance is kept as small as possible [4-6]. The value of $\alpha_{2}$ has been tuned to be less than a critical value which guarantees stability of longitudinal phase space at very low $\alpha_{1}$ values [7]. During the experiment of November 6, 2007, three beams were observed simultaneously rotating in the storage ring with variable transverse sizes, positions, and energies depending on the sextupole strengths and the frequency values of the radio-frequency (rf) cavities [8]. To our knowledge, this was the first experimental evidence of three simultaneously stored beams in an electron storage ring created by direct manipulation of the nonlinear longitudinal beam dynamics. Two beams had already been stored at Brookhaven [9] while two and three beams

\footnotetext{
*maher.attal@sesame.org.jo
}

Published by the American Physical Society under the terms of the Creative Commons Attribution 3.0 License. Further distribution of this work must maintain attribution to the author(s) and the published article's title, journal citation, and DOI. have been stored recently at the Metrology Light Source [10-12].

To understand these experimental observations, the longitudinal equations of motion and the corresponding Hamiltonian have been expanded up to the third-order momentum compaction factor $\alpha_{3}$ as well as taking into account the particle transverse excursion amplitude. This momentum-independent path lengthening term which can be compensated, in principle, by the rf frequency, becomes a more critical parameter at lower $\alpha$ values and its compensation is more challenging for extremely low- $\alpha$ optics. Conditions on the values of $\alpha_{3}$ and the momentumindependent particle path lengthening term have been calculated to explain the behavior and the dynamics of the three simultaneous beams. The analytical investigations and numerical simulations have shown that, by including $\alpha_{3}$, additional stable areas are created in the longitudinal phase space with shape and stability dependent on the value and sign of $\alpha_{3}$. Moreover, some stability criteria valid in the $\alpha_{2}$-dominant longitudinal dynamics become now conditional to the value and sign of $\alpha_{3}$. On the other hand, this raised the importance of controlling $\alpha_{3}$ for the sake of producing stable (very) low- $\alpha$ user operation in the SOLEIL storage ring.

After reporting the three-beam observation in Sec. II, the needed theoretical background to explain the longitudinal beam dynamics is described first in Secs. III and IV. In Sec. III, the expressions of $\alpha_{1}, \alpha_{2}$, and $\alpha_{3}$ are rederived to take into account the momentum-independent path lengthening term, while in Sec. IV, solutions of the longitudinal equations of motion are given for zero and nonzero momentum-independent path lengthening terms. Then Sec. V describes the behavior of the longitudinal phase space under the effects of $\alpha_{1}, \alpha_{2}, \alpha_{3}$, and the momentum-independent path lengthening term. A formula 
for synchrotron frequency as a function of variation in the rf frequency is derived in Sec. VI taking into account the $\alpha_{3}$ and momentum-independent path lengthening terms. This formula is used to evaluate $\alpha_{1}, \alpha_{2}, \alpha_{3}$ from experimental data. In Sec. VII, the behavior of the three beams is explained based on simulation using the Hamiltonian of the longitudinal phase space.

\section{THREE SIMULTANEOUS BEAMS STORED IN THE SOLEIL STORAGE RING}

During a low- $\alpha$ experiment, a mode in which $\alpha_{1}$ theoretical value is $2.1 \times 10^{-5}$ (nominal $\alpha_{1} / 20$ optics), three stable electron beams were simultaneously stored in the SOLEIL storage ring. The beams were imaged using a visible light monitor at a bending magnet exit port [Fig. 1(a)]. The horizontal separation between the two side beams was measured to be $3 \mathrm{~mm}$. The streak camera bunch length measurements gave almost the same rms value of 4 ps for each of the beams at low current. This configuration was stable enough to enable injection directly in the three beams. It was then possible to ramp up the total beam current up to $33 \mathrm{~mA}$ distributed over 104 buckets. The total beam lifetime was $15 \mathrm{~h}$.

By changing the rf-frequency value [Fig. 1(b)] and the strength of one focusing sextupole family [Figs. 1(c) and 1 (d)], densities and transverse distributions of the beams were varying. Migrations of electrons from one rf bucket to another were observed with or without loss of particles depending on the varied physical parameters.
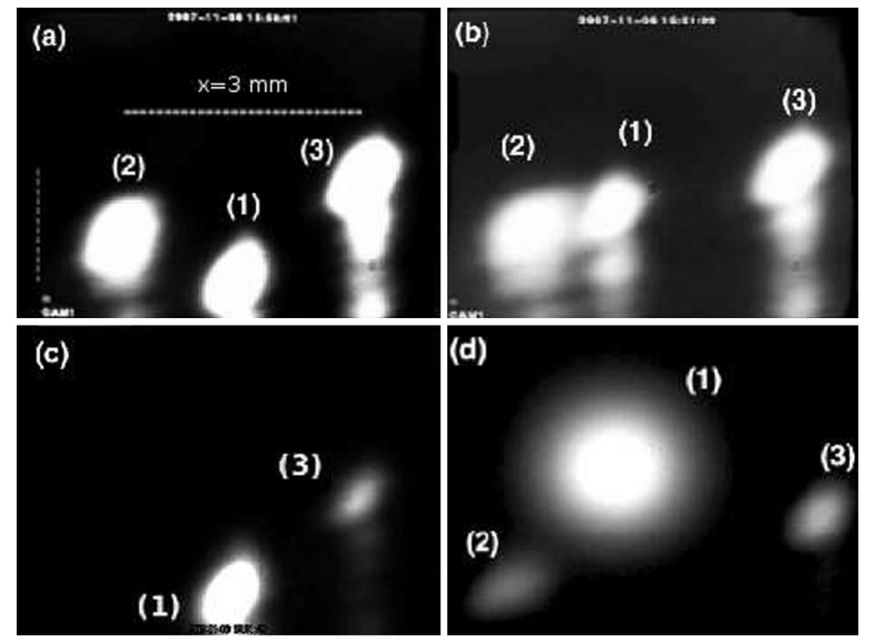

FIG. 1. Different configurations of the three beams stored in the SOLEIL storage ring in November 2007. The four images are obtained using the visible part of a dipole based photon beam for different settings of rf frequency (b) and sextupole magnet strengths $[(\mathrm{c}),(\mathrm{d})]$. Figure axes are vertical and horizontal beam positions. The strong vertical trails of the beams are artifacts of the measurement and result from the mirror quality of the visible light monitor which was damaged after an irreversible thermal stress.
Storing three beams in a circular accelerator can be understood theoretically when higher orders of the momentum compaction factor are included in the model providing that the bucket energy acceptance is large enough and that the chromatic closed orbit of each beam stays within the physical aperture of the accelerator. Phase spaces supporting this event will be given in Sec. VII.

\section{HIGHER ORDER TERMS OF THE MOMENTUM COMPACTION FACTOR}

The momentum compaction factor $\alpha$ is defined by the relative change in the particle path length around a storage ring due to relative deviation in the particle momentum with respect to the synchronous particle, $\alpha=$ $d\left(\Delta L / L_{0}\right)_{\text {chr }} / d \delta$. Mathematically, the $\alpha$ parameter can be developed up to the third order using the relative chromatic variation in path length [7]:

$$
\left(\frac{\Delta L}{L_{0}}\right)_{\mathrm{chr}}=\alpha_{1} \delta+\alpha_{2} \delta^{2}+\alpha_{3} \delta^{3}
$$

where $L_{0}$ is the length of the synchronous particle orbit and $\delta=\Delta p / p_{0}$ is the relative momentum deviation from the synchronous particle momentum $p_{0}$. Equivalently, the variation in path length can be expanded to the third order as

$$
\frac{\Delta L}{L_{0}}=\frac{1}{L_{0}} \int_{0}^{L_{0}}\left[\frac{1-k_{x} x}{2}\left(x^{\prime 2}+y^{\prime 2}\right)+k_{x} x\right] d s,
$$

where $x(y)$ and $x^{\prime}\left(y^{\prime}\right)$ are respectively the horizontal (vertical) electron position and angle, $k_{x}=1 / \rho_{x}$ where $\rho_{x}$ is the horizontal curvature radius of the bending magnet, taking into account that $x^{\prime}, y^{\prime}, k_{x} x$ are much less than unity [7].

By breaking down the horizontal particle amplitude (angle) $x\left(x^{\prime}\right)$ into betatron oscillation term $x_{\beta}\left(x_{\beta}^{\prime}\right)$, residual closed orbit term $x_{0}\left(x_{0}^{\prime}\right)$, and off-momentum orbit term $D_{x} \delta\left(D_{x}^{\prime} \delta\right)$ expanded to third order in $\delta$, then one gets

$$
\begin{gathered}
x=x_{\beta}+x_{0}+D_{x 1} \delta+D_{x 2} \delta^{2}+D_{x 3} \delta^{3}, \\
x^{\prime}=x_{\beta}^{\prime}+x_{0}^{\prime}+D_{x 1}^{\prime} \delta+D_{x 2}^{\prime} \delta^{2}+D_{x 3}^{\prime} \delta^{3},
\end{gathered}
$$

where $D_{x 1}\left(D_{x 1}^{\prime}\right), D_{x 2}\left(D_{x 2}^{\prime}\right)$, and $D_{x 3}\left(D_{x 3}^{\prime}\right)$ are respectively the horizontal first-, second-, and third-order dispersion function terms (and their respective derivatives). Similar expressions can be derived for the vertical plane assuming that the vertical dispersion function is negligible:

$$
y=y_{\beta}+y_{0}, \quad y^{\prime}=y_{\beta}^{\prime}+y_{0}^{\prime},
$$

where $y_{\beta}\left(y_{\beta}^{\prime}\right)$ and $y_{0}\left(y_{0}^{\prime}\right)$ are respectively the vertical betatron and the residual closed orbit amplitude (divergence) of the beam.

By inserting Eqs. (3) and (4) into Eq. (2) and considering the terms up to third order in energy deviation $\delta$, the total relative variation in path length becomes [4] 


$$
\begin{aligned}
\frac{\Delta L}{L_{0}}= & \frac{1}{L_{0}} \int_{0}^{L_{0}}\left[\epsilon+\left(k_{x} D_{x 1}-k_{x} D_{x 1} \epsilon\right) \delta\right. \\
& +\left(\frac{D_{x 1}^{\prime 2}}{2}+k_{x} D_{x 2}-k_{x} D_{x 2} \epsilon\right) \delta^{2} \\
& \left.+\left(D_{x 1}^{\prime} D_{x 2}^{\prime}+k_{x} D_{x 3}-\frac{k_{x} D_{x 1}^{\prime 2} D_{x 1}}{2}-k_{x} D_{x 3} \epsilon\right) \delta^{3}\right] d s,
\end{aligned}
$$

where $\epsilon=\left(x_{\beta}^{\prime 2}+x_{0}^{\prime 2}+y_{\beta}^{\prime 2}+y_{0}^{\prime 2}\right) / 2$ represents the contributions of betatron oscillations and residual closed orbit to the relative variation of the path length. In deriving Eq. (5), all the linear terms in $x_{\beta}, x_{\beta}^{\prime}, y_{\beta}$, and $y_{\beta}^{\prime}$ vanish since betatron amplitudes and angles average to zero after many turns [3]. All the linear terms in $x_{0}, x_{0}^{\prime}, y_{0}$, and $y_{0}^{\prime}$ can also be dropped when the closed orbit is corrected such as the residual distortion averages to zero. Moreover, since betatron oscillations and closed orbit distortion are independent, the cross terms of these variables also vanish [7].

By comparing Eqs. (1) and (5), one deduces an expression for the momentum-independent term in the path length variation, $\chi$, which represents path lengthening effects of betatron oscillations and closed orbit errors:

$$
\chi=\frac{1}{L_{0}} \int_{0}^{L_{0}} \epsilon d s,
$$

and expressions for momentum compaction factor terms up to the third order:

$$
\begin{aligned}
& \alpha_{1}=\frac{1}{L_{0}} \int_{0}^{L 0} k_{x} D_{x 1}(1-\epsilon) d s, \\
& \alpha_{2}=\frac{1}{L_{0}} \int_{0}^{L 0}\left[\frac{D_{x 1}^{\prime 2}}{2}+k_{x} D_{x 2}(1-\epsilon)\right] d s, \\
& \alpha_{3}=\frac{1}{L_{0}} \int_{0}^{L 0}\left[D_{x 1}^{\prime} D_{x 2}^{\prime}-\frac{k_{x} D_{x 1} D_{x 1}^{\prime 2}}{2}+k_{x} D_{x 3}(1-\epsilon)\right] d s .
\end{aligned}
$$

To give an order of magnitude for the parameter $\chi$, this latter can be split into two main contributions: betatron oscillation and orbit distortion, which gives in more practical units [7], $\chi \approx\left(\epsilon_{x}\left\langle\gamma_{x}\right\rangle+\epsilon_{y}\left\langle\gamma_{y}\right\rangle\right) / 4+\left(\left\langle x_{0}^{\prime 2}\right\rangle+\left\langle y_{0}^{\prime 2}\right\rangle\right) / 2$ where $\epsilon_{x}\left(\epsilon_{y}\right)$ is the horizontal (vertical) emittance and $\gamma_{x}\left(\gamma_{y}\right)$ is one of the Twiss parameters. In the low- $\alpha$ optics of SOLEIL, the horizontal emittance is $\epsilon_{x}=8.5 \times$ $10^{-9} \mathrm{mrad}$ and the Twiss parameter average value is $\left\langle\gamma_{x}\right\rangle \approx 2$ (as given by BETA code [13]). By neglecting the much smaller vertical term, the value of betatron oscillation part is $4.3 \times 10^{-9}$, which is the minimum value of $\chi$. In the normal operational optics of SOLEIL the residual orbit peaks are $\pm 0.3 \mathrm{~mm}$. However, due to the higher sensitivity to energy fluctuation of the orbit distortion at lower $\alpha$ value, the residual orbit peaks will become larger. Simulations using BETA code showed that orbits with residual peaks of $\pm 0.2 \mathrm{~mm}, \pm 1 \mathrm{~mm}$, and $\pm 5 \mathrm{~mm}$ corre- spond to the values $1.3 \times 10^{-9}, 4 \times 10^{-8}$, and $1 \times 10^{-6}$, respectively, for the closed orbit part of $\chi$. Although the rf frequency can be used to compensate for the orbit distortion, the substantially increasing sensitivity of lower $\alpha$ optics to energy fluctuations or horizontal orbit deviations makes it worth including the $\chi$ effect in the longitudinal phase-space dynamics.

For further use in the next section, the relation between momentum compaction factor and phase-slip factor will be derived up to the third order. The later is derived in a similar way using the relative variation in the particle chromatic revolution time up to third order in $\delta$ [14]. Using the relation between the variations in path length and the corresponding revolution time $[15,16]$, one finds [4]

$$
\begin{aligned}
& \eta_{1}=\alpha_{1}-\frac{1}{\gamma^{2}}, \\
& \eta_{2}=\alpha_{2}+\frac{1}{2 \gamma^{4}}+\frac{3 \beta^{2}}{2 \gamma^{2}}, \\
& \eta_{3}=\alpha_{3}-\frac{1}{2 \gamma^{6}}-\frac{3 \beta^{2}}{2 \gamma^{4}}-\frac{2 \beta^{4}}{\gamma^{2}},
\end{aligned}
$$

where $\eta_{1}, \eta_{2}$, and $\eta_{3}$ are the first-, second-, and third-order phase-slip factors, $\beta=v_{0} / c=\sqrt{1-1 / \gamma^{2}}$ is the synchronous particle velocity $v_{0}$ normalized to the speed of light $c$, and $\gamma$ is the Lorentz factor defined as the ratio of the particle energy $E_{0}$ to its rest mass energy $E_{m}$.

Taking into account that the Lorentz factor for the $2.75 \mathrm{GeV}$ SOLEIL storage ring is of 5382, the previous formula can be simplified (highly relativistic case): all energy-dependent slip-factor terms are negligible $\left(\eta_{k} \approx \alpha_{k}\right.$ for $\left.k=1,2,3\right)$.

\section{SOLUTIONS OF THE LONGITUDINAL EQUATIONS OF MOTION INCLUDING $\alpha_{3}$ TERM}

The equations of longitudinal motion, expanded to third order in phase-slip factor, can be written compactly as follows $[4,17]$ :

$$
\begin{gathered}
\frac{d \psi}{d t}=\omega_{\mathrm{rf}}\left(\chi+\eta_{1} \delta+\eta_{2} \delta^{2}+\eta_{3} \delta^{3}\right), \\
\frac{d \delta}{d t}=\frac{e V_{R F}}{\beta^{2} E_{0} T_{0}}\left(\sin \Phi-\sin \Phi_{s}\right),
\end{gathered}
$$

where $\omega_{\mathrm{rf}}=2 \pi h f_{0}$ is the rf-angular frequency, $h$ the harmonic number, $f_{0}$ is the nominal particle revolution frequency, $\psi=\Phi-\Phi_{s}$ is the relative phase between the particle phase $\Phi$ and the synchronous phase $\Phi_{s}, V_{\mathrm{rf}}$ is the total $\mathrm{rf}$ voltage in the storage ring, and $T_{0}$ is the synchronous particle revolution time.

The Hamiltonian describing longitudinal phase space can be constructed by integrating Eqs. (9) and (10) using $\psi$ and $\delta$ as canonically conjugate variables: 
TABLE I. Main experimental parameters for the nominal optics of the SOLEIL storage ring operation in 2007 as calculated with the BETA code.

\begin{tabular}{lcc}
\hline \hline Parameter (unit) & Symbol & Value \\
\hline Energy $(\mathrm{GeV})$ & $E_{0}$ & 2.75 \\
Frequency $(\mathrm{MHz})$ & $f_{\mathrm{rf}}$ & 352.202 \\
Harmonic number & $h$ & 416 \\
rf voltage (MV) & $V_{\mathrm{rf}}$ & 2.4 \\
Revolution frequency $(\mu \mathrm{s})$ & $T_{0}$ & 1.181 \\
Synchronous phase (degree) & $\Phi_{s}$ & 156.8 \\
1st momentum compaction factor & $\alpha_{1}$ & $4.38 \times 10^{-4}$ \\
2nd momentum compaction factor & $\alpha_{2}$ & $4.45 \times 10^{-3}$ \\
3rd momentum compaction factor & $\alpha_{3}$ & $-3.95 \times 10^{-3}$ \\
\hline \hline
\end{tabular}

$$
\begin{aligned}
H= & \omega_{\mathrm{rf}}\left(\chi \delta+\frac{\eta_{1}}{2} \delta^{2}+\frac{\eta_{2}}{3} \delta^{3}+\frac{\eta_{3}}{4} \delta^{4}\right) \\
& +\frac{e V_{\mathrm{rf}}}{\beta^{2} E_{0} T_{0}}\left[\cos \Phi-\cos \Phi_{s}+\left(\Phi-\Phi_{s}\right) \sin \Phi_{s}\right],
\end{aligned}
$$

Since only the highly relativistic case is considered, the above approximations $\eta_{1} \approx \alpha_{1}, \eta_{2} \approx \alpha_{2}, \eta_{3} \approx \alpha_{3}$, and $\beta \approx 1$ will be used from now on.

By solving Eqs. (9) and (10) for fixed phases and momentum deviations, one can determine the coordinates $(\Phi, \delta)$ of the stable and unstable fixed points in longitudinal phase space [18]. Phases of the fixed points are given by the two solutions of Eq. (10), $\Phi=\Phi_{s}$ and $\Phi=\pi-\Phi_{s}$. In the linear case, the rf-bucket layer will be centered on the synchronous phase $\Phi_{s}$ with the unstable fixed points centered at $\pi-\Phi_{s}$ in the phase range $[0,2 \pi]$.

Momentum deviations of those fixed points can be obtained by solving Eq. (9) for $\delta$ :

$$
\alpha_{3} \delta^{3}+\alpha_{2} \delta^{2}+\alpha_{1} \delta+\chi=0 .
$$

Solutions of the third-order Eq. (12) can be real or complex depending on the values of $\alpha_{1}, \alpha_{2}, \alpha_{3}$, and $\chi$. The real solutions give the number of stable bucket layers in longitudinal phase space whereas complex solutions indicate that the corresponding bucket layers do not exist in the phase space. Two cases are illustrated hereafter using the nominal optics (see Table I); the effect of $\chi$ is taken into account in the second case.

\section{A. Case of a negligible momentum-independent term $\boldsymbol{X}$}

In this case, the closed orbit is very well corrected so that $\chi$ can be negligible ( $\mathrm{rf}$ frequency can theoretically compensate for $\chi$ ). Equation (12) simplifies to

$$
\delta\left(\alpha_{3} \delta^{2}+\alpha_{2} \delta+\alpha_{1}\right)=0,
$$

with the solutions

$$
\delta_{1}=0, \quad \delta_{2,3}=\frac{-\alpha_{2} \pm \sqrt{\alpha_{2}^{2}-4 \alpha_{3} \alpha_{1}}}{2 \alpha_{3}} .
$$

Using the SOLEIL nominal operational parameters (see Table I), the numerical solutions are $\left\{\delta_{1}=0, \delta_{2}=\right.$ $\left.-0.091, \delta_{2}=1.22\right\}$. These three real solutions indicate the existence of one on-momentum bucket layer in longitudinal phase space with coordinates $\left(\Phi_{1}=\Phi_{s}, \delta_{1}=0\right)$, i.e., the already stored electron beam, and two offmomentum beams with coordinates $\left(\Phi_{2}=\pi-\Phi_{s}, \delta_{2}=\right.$ $-0.091)$, and $\left(\Phi_{3}=\pi-\Phi_{s}, \delta_{3}=1.22\right)$. However, these off-momentum beams are out of the machine transverse momentum and physical acceptances so no electron can be stored in them.

\section{B. Case of a non-negligible momentum-independent term $\chi$}

When the residual orbit distortion is non-negligible (for instance, the resolution of the $352 \mathrm{MHz}$ master oscillator can limit the compensation of the $\chi$ term using the $\mathrm{rf}$ frequency), solutions of Eq. (12) can be nonetheless derived using the handbook of mathematical functions [19]:

$$
\begin{aligned}
\delta_{1} & =-\frac{\alpha_{2}}{3 \alpha_{3}}-(\zeta-v), \\
\delta_{2,3} & =-\frac{\alpha_{2}}{3 \alpha_{3}}+\frac{1}{2}(\zeta-v) \pm \frac{i \sqrt{3}}{2}(\zeta+v),
\end{aligned}
$$

where $\zeta=2^{1 / 3}\left(3 \alpha_{1} \alpha_{3}-\alpha_{2}^{2}\right) /\left(3 \alpha_{3} \xi\right), v=\xi /\left(32^{1 / 3} \alpha_{3}\right)$, and $\xi=\left(\hat{\xi}+\sqrt{4\left(3 \alpha_{1} \alpha_{3}-\alpha_{2}^{2}\right)^{3}+\hat{\xi}^{2}}\right)^{1 / 3}$ with $\hat{\xi}=$ $9 \alpha_{1} \alpha_{2} \alpha_{3}-2 \alpha_{2}^{3}-27 \alpha_{3}^{2} \chi$.

Using again the SOLEIL parameters of the nominal optics, three bucket layers exist in the longitudinal phase space as long as $\chi$ is less than $1 \times 10^{-5}$, i.e., the residual orbit peaks are less than $\pm 50 \mathrm{~mm}$ assuming linear scaling. Even for this critical value of the $\chi$ parameter, only one bucket is accepted by transverse beam dynamics and physical aperture of the storage ring; however, its energy is shifted $\left(\delta_{1}=-0.0380\right)$. For the two other buckets, the energy deviations are too large $\left(\delta_{2}=-0.0547, \delta_{3}=\right.$ 1.2192).

Beyond this critical value, only one bucket layer survives in the longitudinal phase space but with large energy deviation that is well beyond the transverse dynamics and transverse physical acceptances. For example, for $\chi=1.1 \times 10^{-5}$, the fixed points are found to be $\left(\delta_{1}=-0.0464+0.0114 i, \quad \delta_{2}=-0.0464-0.0114 i\right.$, $\left.\delta_{3}=1.2194\right)$.

The very large value of $\chi$ used in this case is not practical for the storage ring operation. The aim is just to exhibit its effect on the longitudinal phase space using the less sensitive nominal optics to such distortion. However, it will be shown that its value becomes more critical for optics with extremely low $\alpha$ values. The optics becomes highly sensitive to energy and orbit fluctuations so that a small value of $\chi$ can cause beam loss. At such conditions compensating for the $\chi$ value by using the $\mathrm{rf}$ frequency may become very challenging. 


\section{LONGITUDINAL BEAM DYNAMICS AND PHASE SPACE}

The aim of this section is to establish a theoretical background which will be used to explain the three-beam event. For this purpose the Hamiltonian describing the dynamics of the longitudinal phase space including the effects of $\chi, \alpha_{1}, \alpha_{2}$, and $\alpha_{3}$ values [see Eq. (12)] is written as

$$
\begin{aligned}
H= & \omega_{\mathrm{RF}}\left(\chi \delta+\frac{\alpha_{1}}{2} \delta^{2}+\frac{\alpha_{2}}{3} \delta^{3}+\frac{\alpha_{3}}{4} \delta^{4}\right) \\
& +\frac{e V_{\mathrm{RF}}}{E_{0} T_{0}}\left[\cos \Phi-\cos \Phi_{s}+\left(\Phi-\Phi_{s}\right) \sin \Phi_{s}\right]
\end{aligned}
$$

The effects of the $\chi, \alpha_{1}$, and $\alpha_{2}$ values on the beam dynamics will be first investigated without taking into account $\alpha_{3}$ to show in a last part how this latter reshapes the phase space. These investigations will be done first using the nominal optics, and then applied to the low- $\alpha$ optics.

\section{A. Effect of the momentum-independent term $\boldsymbol{\chi}$}

By increasing the $\chi$ parameter from the initial betatron oscillation part, the nominal phase space starts to get distorted. The deformation is featured by shifting energy of the on-momentum rf-bucket layer by the value $-\Delta \delta_{1}^{\text {shift }}$ while shifting that of the second off-momentum rf-bucket layer by the value $\Delta \delta_{2}^{\text {shift }}$. Moreover, the bucket momentum
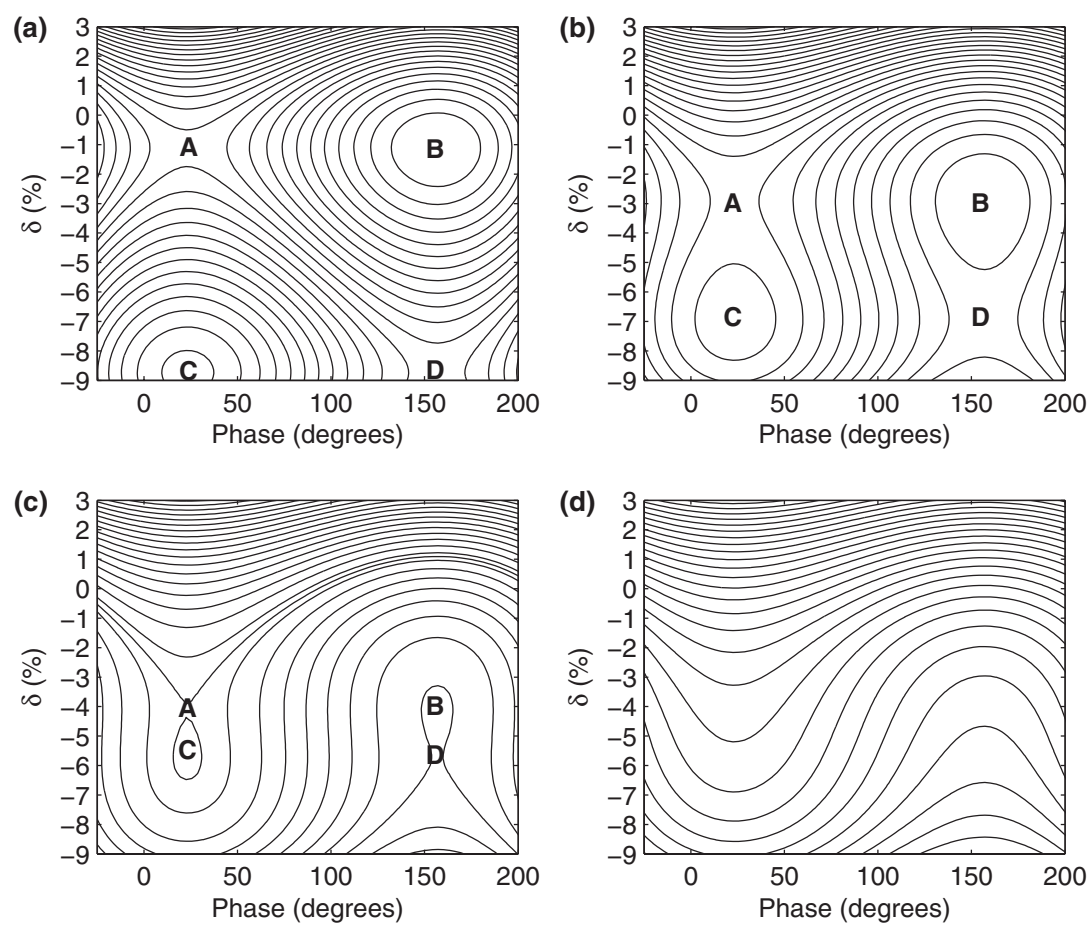

TABLE II. Coordinates of the stable fixed points in function of the $\chi$ value for the first and second bucket series of Fig. 2.

\begin{tabular}{lcc}
\hline \hline$\chi$ value & B coordinates & C coordinates \\
\hline $4.30 \times 10^{-9}$ & $\left(\Phi_{s},+0.0000\right)$ & $\left(\pi-\Phi_{s},-0.0984\right)$ \\
$9.00 \times 10^{-6}$ & $\left(\Phi_{s},-0.0292\right)$ & $\left(\pi-\Phi_{s},-0.0692\right)$ \\
$1.05 \times 10^{-6}$ & $\left(\Phi_{s},-0.0413\right)$ & $\left(\pi-\Phi_{s},-0.0571\right)$ \\
$1.30 \times 10^{-5}$ & Undefined & Undefined \\
\hline \hline
\end{tabular}

acceptance starts to get deformed. This is shown in Fig. 2 displaying the impact of the $\chi$ value on the longitudinal phase space of SOLEIL for the nominal optics (Table I). At larger $\chi$ values the rf buckets turn into alpha buckets [15] with smaller areas, and then no stable points exist in the phase space, so that no beam can be stored in the storage ring (in Fig. $2 \alpha_{3}$ is set to zero).

The marks $\mathrm{A}$ and $\mathrm{B}$ represent respectively unstable, stable fixed points in the first bucket layer while C, D are respectively stable, unstable fixed points in the second bucket layer. Coordinates of the stable fixed points B and $\mathrm{C}$ are listed in Table II. It is worth mentioning that $\Delta \delta_{1}^{\text {shift }}=\chi / \alpha_{1}$ when the following condition is met: $\alpha_{2} \ll \alpha_{1}$.

The case of Fig. 2(d) can be seen clearly from the solutions of Eq. (12) when $\alpha_{3}=0, \delta_{1,2}=\left(-\alpha_{1} \pm\right.$ $\left.\sqrt{\alpha_{1}^{2}-4 \alpha_{2} \chi}\right) / 2 \alpha_{2}$, where increasing the value of $\chi$ will make, at some point, all the solutions complex.

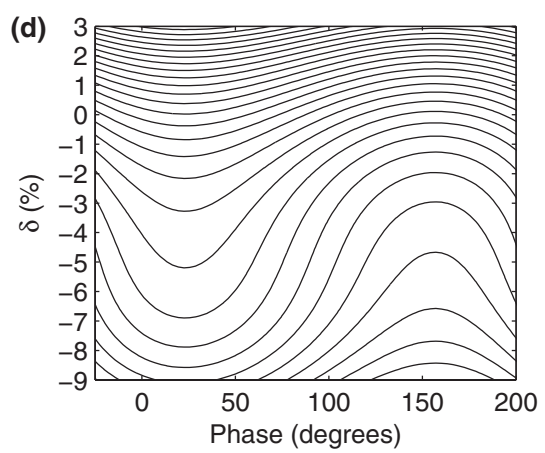

FIG. 2. Effect of the $\chi$ parameter on the longitudinal phase space for the SOLEIL nominal optics with $\alpha_{1}=4.38 \times 10^{-4}, \alpha_{2}=$ $4.45 \times 10^{-3}$ assuming $\alpha_{3}=0$, and $\chi=4.3 \times 10^{-9}$ in (a), $\chi=9 \times 10^{-6}$ in (b), $\chi=1.05 \times 10^{-5}$ in (c), and $\chi=1.3 \times 10^{-5}$ in (d). The fixed points B and D are located at phase $\Phi_{s}$, while the points A and C are located at phase $\pi-\Phi_{s}$. The on-momentum bucket is progressively off-centered while $\chi$ increases (see Table II for numerical values of the stable fixed points). 

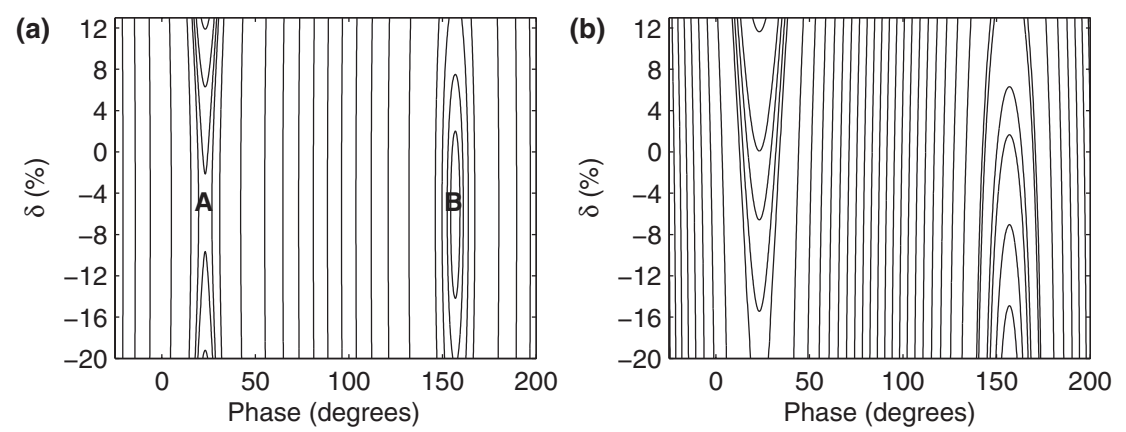

FIG. 3. Impact of the $\chi$ parameter on the longitudinal phase space for very low- $\alpha$ optics with $\alpha_{1}=1 \times 10^{-7}, \alpha_{2}=1 \times 10^{-7}$ assuming $\alpha_{3}=0$, and $\chi=5.5 \times 10^{-9}$ in (a), $\chi=3 \times 10^{-8}$ in (b) which correspond respectively to an orbit distortion of \pm 0.2 and $\pm 1 \mathrm{~mm}$. The fixed points marked by A (unstable) and B (stable) in case (a) are not defined anymore in case (b).

As stressed before, the $\chi$ parameter can have a critical effect on the longitudinal phase space for extremely low- $\alpha$ optics. Figure 3 displays this feature for a very low- $\alpha$ optics of SOLEIL with $\alpha_{1}=1 \times 10^{-7}$. Figure 3(a) shows that a value of $\chi=5.5 \times 10^{-9}$ (i.e. orbit distortion with maximum peaks of $\pm 0.2 \mathrm{~mm}$ ) shifts the energy of the onmomentum beam (B) out of the machine transverse momentum acceptance with an offset larger than $-5 \%$. A value of $\chi=3 \times 10^{-8}$ (i.e. orbit distortion with maximum peaks of $\pm 1 \mathrm{~mm}$ ) is already large enough to make all the buckets shrink to zero momentum acceptance as shown in Fig. 3(b).

As a result, correcting the closed orbit is essential to get the beam stored at very low- $\alpha$ optics.
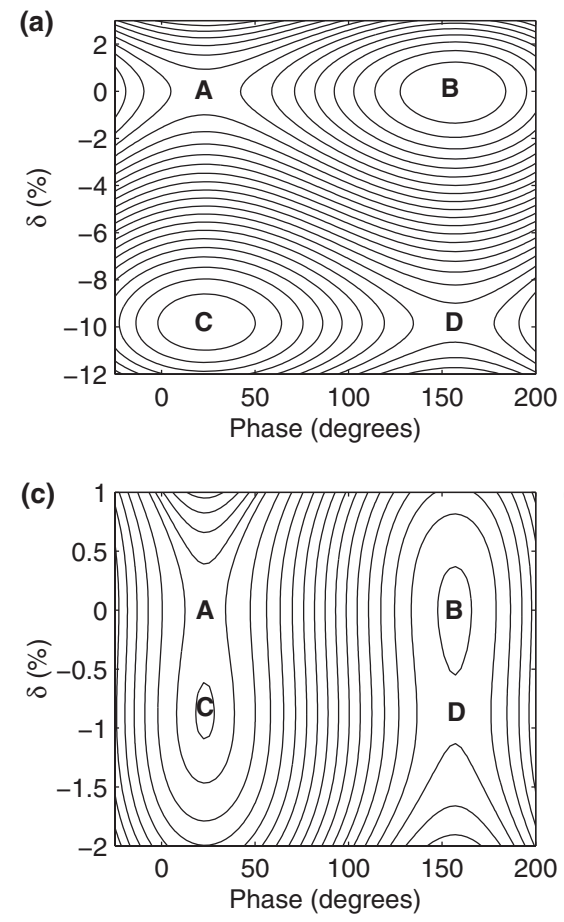

\section{B. Effect of the first-order momentum compaction factor}

Equation (16) shows that the $\alpha_{1}$ parameter supports the symmetry of the linear rf buckets around the $\Phi$ axis as well as the linear behavior of the longitudinal phase space. The negative sign of $\alpha_{1}$ reverses the phase space around the $\Phi=90^{\circ}$ axis so that the stable and unstable fixed points exchange their positions.

\section{Effect of the second-order momentum compaction factor $\alpha_{2}$}

The second-order momentum compaction factor $\alpha_{2}$ has an effect similar to that of $\chi$ in terms of breaking down the
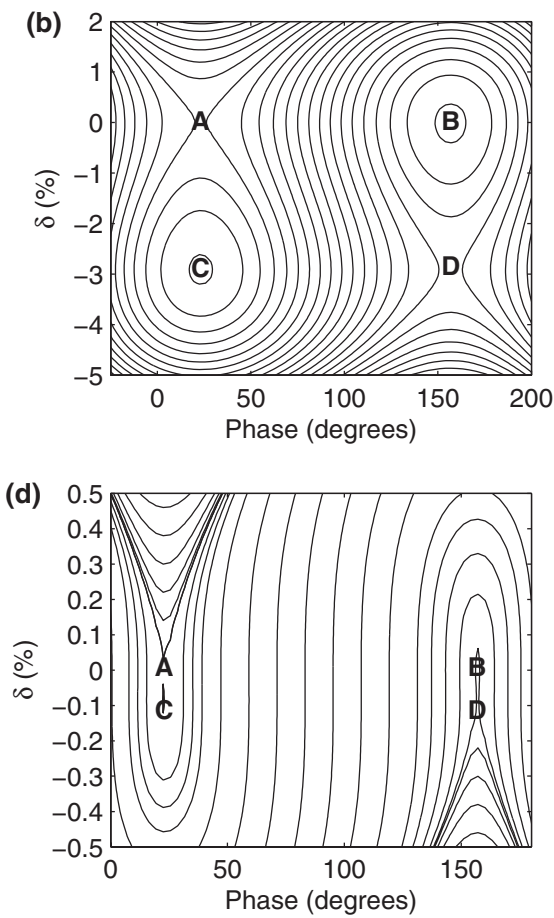

FIG. 4. Effect of the $\alpha_{2}$ on the longitudinal phase space for the SOLEIL using the nominal value for $\alpha_{1}=4.38 \times 10^{-4}$ assuming $\chi=4.3 \times 10^{-9}$ and $\alpha_{3}=0$. The energy gap between the two rf-bucket layers decreases while the momentum acceptance (i.e. areas) of the buckets shrinks to zero as $\alpha_{2}$ increases from $4.45 \times 10^{-3}$ in (a) to $1.5 \times 10^{-2}$ in (b), $5 \times 10^{-2}$ in (c), and $5 \times 10^{-1}$ in (d). No energy shift occurs in contrast to the effect of the $\chi$ parameter. The stable and unstable fixed points are respectively (A, D) and (B, C). 

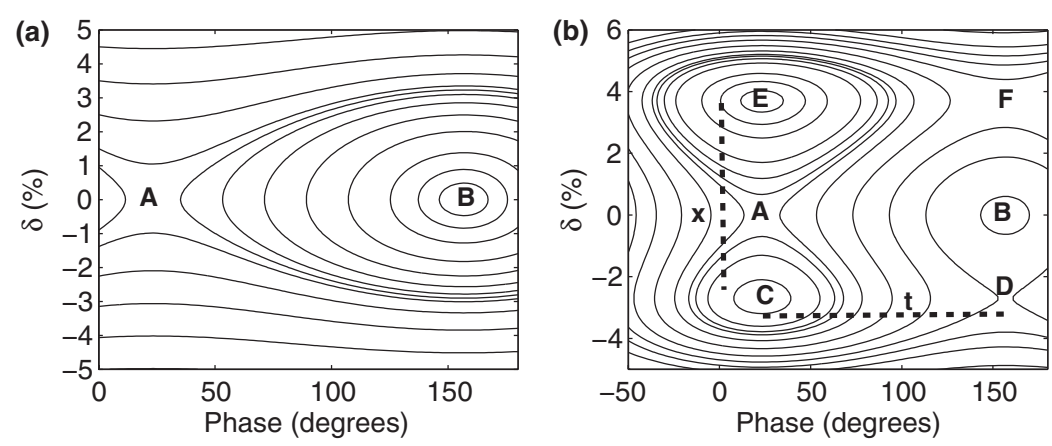

FIG. 5. Effect of the sign of $\alpha_{3}$ on the longitudinal phase space for the nominal SOLEIL optics with $\alpha_{1}=4.4 \times 10^{-4}, \alpha_{2}=$ $4.5 \times 10^{-3}$, and the values $\alpha_{3}=0.39$ in (a), $\alpha_{3}=-0.39$ in (b), assuming the minimum value $\chi=4.3 \times 10^{-9}$. Three beams can be stored in case (b) providing that the transverse dynamic and physical acceptances are large enough. The transverse distance $x$ between off-momentum stable buckets is $D_{x}\left(\delta_{E}\right)-D\left(\delta_{C}\right)$ while $t$ is the time distance between buckets at the different phases. The letters A, D, $\mathrm{F}$ represent the unstable fixed points and $\mathrm{B}, \mathrm{C}$, and $\mathrm{F}$, the stable fixed points.

symmetry of rf-bucket momentum acceptance, reducing the bucket sizes and reducing the energy gaps between the on-momentum and off-momentum bucket layers. However, it does not cause energy shift to the onmomentum one. This can be seen from Fig. 4 where $\alpha_{2}$ is increased from the nominal value, $\alpha_{2}=4.45 \times 10^{-3}$, to respectively $1.5 \times 10^{-2}, 5 \times 10^{-2}$, and 0.5 . Other parameters are kept constant: $\alpha_{1}=4.38 \times 10^{-4}, \chi=$ $4.3 \times 10^{-9}$, and $\alpha_{3}=0$.

The vertical distance between the two rf-bucket layers in Fig. 4 is given by [15,20]: $\delta_{\text {disp }}=-\alpha_{1} / \alpha_{2}$.

According to Eq. (12), with $\alpha_{3}=0$, no stable fixed point can be found in the phase space if the condition $\alpha_{2}>$ $\alpha_{1}^{2} / 4 \chi$ is fulfilled. So for low- $\alpha$ optics, $\alpha_{2}$ must be minimized to keep enough bucket momentum acceptance for reasonable injection efficiency and beam lifetime. Moreover, if the energy gap between the two bucket layers stays within the machine transverse energy acceptance and the physical aperture, then two simultaneous beams are expected in the storage ring. It is worth mentioning that the negative sign of $\alpha_{2}$ reverses the phase space around the $\delta=0$ axis so that the off-momentum bucket layers exchange their positions.

\section{Effect of the third-order momentum compaction factor $\alpha_{3}$}

By taking into account the third order of the momentum compaction factor, $\alpha_{3}$, the dynamics described in Secs. VA and V C will display different features; there still would be one bucket layer in the phase space with finite energy acceptance regardless of the values of $\chi$ and $\alpha_{2}$, however shifted in energy by a value that may exceed the machine transverse energy acceptance. This can be seen from the solutions of Eq. (12) when $\alpha_{3} \neq 0$. In the case $\alpha_{1} \alpha_{3}>0$, the phase-space symmetry around the $\phi$ axis is enhanced, depending on the value of $\alpha_{3}$. On the contrary, if the condition $\alpha_{1} \alpha_{3}<0$ is met, the energy gaps between the on-momentum bucket layer and both the positive and negative off-momentum ones are reduced depending on the values of $\alpha_{1}$ and $\alpha_{2}$. For example, using the values of the nominal SOLEIL optics three bucket layers are within the transverse machine acceptance of $4 \%$ as long as $\alpha_{3} \leq-0.39$.

Figure 5 shows the impact of the sign of $\alpha_{3}$, with different signs, on SOLEIL longitudinal phase space using SOLEIL nominal parameters $\alpha_{1}=4.4 \times 10^{-4}, \alpha_{2}=$ $4.5 \times 10^{-3}$, and $\chi=4.3 \times 10^{-9}$. Figure 5(b) exhibits a phase-space portrait obtained with a negative value of $\alpha_{3}$ where three bucket layers simultaneously exist: one with nominal energy and two off-momentum buckets with energy offsets $+4.0 \%$ and $-2.8 \%$. The on-momentum $\mathrm{rf}$ bucket is separated from the off-momentum ones longitudinally by $\left|\pi-2 \Phi_{s}\right|$ in phase or by $t=1.054 \mathrm{~ns}$ in time according to SOLEIL rf parameters (Table I). In the horizontal plane, the off-momentum buckets are separated by a distance " $\mathrm{x}$ " depending on the value of dispersion function $D_{x}$ at the observation location. Since the key parameter $\alpha_{3}$ governs the possibility to store or not three simultaneous beams then for more stable low- $\alpha$ operation in the synchrotron SOLEIL, it needs to be controlled with great care.

\section{SYNCHROTRON FREQUENCY INCLUDING $\alpha_{3}$ TERM}

At small synchrotron oscillation amplitude, the synchrotron frequency $f_{s}$ is directly proportional to the square root of $\alpha$ [21]:

$$
f_{s}=f_{0} \sqrt{\frac{h e V_{\mathrm{rf}} \cos \Phi_{s}}{2 \pi E_{0}}} \alpha^{\frac{1}{2}}
$$

where $E_{0}$ and $f_{0}$ are the energy and the revolution frequency of the synchronous particle, respectively.

When the high order terms of $\alpha$ are taken into account, Eq. (17) becomes 


$$
f_{s}=f_{0} \sqrt{\frac{h e V_{\mathrm{rf}} \cos \Phi_{s}}{2 \pi E_{0}}}\left(\alpha_{1}+2 \alpha_{2} \delta+3 \alpha_{3} \delta^{2}\right)^{\frac{1}{2}}
$$

Writing Eq. (18) in terms of the solutions of Eq. (15) results in three different synchrotron frequencies corresponding to each bucket layer [4]:

$$
f_{s k}=f_{0} \sqrt{\frac{h e V_{\mathrm{rf}}\left|\cos \Phi_{s}\right|}{2 \pi E_{0}\left(1+\delta_{k}\right)}}\left(\alpha_{1}+2 \alpha_{2} \delta_{k}+3 \alpha_{3} \delta_{k}^{2}\right)^{\frac{1}{2}},
$$

with $k=1,2,3$. The absolute value for $\cos \Phi_{s}$ comes from the fact that the buckets are centered at two phases $\Phi_{s}$ and $\pi-\Phi_{s}$ having the same cosine values but with opposite signs.

The most direct way to evaluate $\alpha_{1}, \alpha_{2}$, and $\alpha_{3}$ is to measure the synchrotron frequency of any of the three beams as a function of relative variation in $\mathrm{rf}$ frequency $\Delta f_{\text {rf }} / f_{\text {rf }}[9,11,21]$.

To establish the required function for the on-momentum beam, for instance, the corresponding energy deviation $\delta_{1}$ is considered. Assuming a well-corrected closed orbit, the energy deviation $\delta_{1}$ is so small that it can be neglected in the denominator of Eq. (19) and the beam energy can be approximated to the nominal one without loss of accuracy:

$$
f_{s 1}=f_{0} \sqrt{\frac{h e V_{\mathrm{rf}}\left|\cos \Phi_{s}\right|}{2 \pi E_{0}}}\left(\alpha_{1}+2 \alpha_{2} \delta_{1}+3 \alpha_{3} \delta_{1}^{2}\right)^{1 / 2}
$$

Inserting the $\delta_{1}$ value from Eq. (15) leads, after some mathematical manipulations, to [4]

$$
\begin{aligned}
f_{s 1}(\chi) \approx & f_{0} \sqrt{\frac{h e V_{\mathrm{rf}}\left|\cos \Phi_{s}\right|}{2 \pi E_{0}}}\left(-\alpha_{1}+\frac{\alpha_{2}^{2}}{3 \alpha_{3}}\right. \\
& \left.+\frac{2^{2 / 3}\left(3 \alpha_{1} \alpha_{3}-\alpha_{2}^{2}\right)^{2}}{3 \alpha_{3} \xi^{2}}+\frac{\xi^{2}}{2^{2 / 3} 3 \alpha_{3}}\right)^{1 / 2}
\end{aligned}
$$

with $\xi=\left[\varpi+\sqrt{4\left(3 \alpha_{1} \alpha_{3}-\alpha_{2}^{2}\right)^{3}+\varpi^{2}}\right]^{1 / 3}$ and $\varpi=$ $9 \alpha_{1} \alpha_{2} \alpha_{3}-2 \alpha_{2}^{3}-27 \alpha_{3}^{2} \frac{\Delta f_{\mathrm{rf}}}{f_{\mathrm{rf}}}$ where the relation $\chi \approx$ $\Delta f_{\text {rf }} / f_{\text {rf }}[21]$ has been used.

It is worth mentioning that when $\alpha_{3}$ is not taken into account, Eq. (21) will be reduced to the known secondorder formula: $f_{s}=f_{0} \sqrt{\frac{h e V_{\mathrm{rf}} \cos \Phi_{s}}{2 \pi E_{0}}} \alpha_{1}^{1 / 2}\left(1-4 \frac{\alpha_{2}}{\alpha_{1}^{2}} \frac{\Delta f_{\mathrm{rf}}}{f_{\mathrm{rf}}}\right)^{1 / 4}$ $[9,20,21]$.

This method was applied at SOLEIL to evaluate experimentally the values of $\alpha_{1}, \alpha_{2}$, and $\alpha_{3}$ in one of the low- $\alpha$ optics mode whose theoretical parameters are $\alpha_{1}=$ $4.35 \times 10^{-6}, \alpha_{2}=-1.81 \times 10^{-5}$, and $\alpha_{3}=7.5 \times 10^{-2}$. By measuring the synchrotron frequency $f_{s}$ as a function of $\Delta f_{\text {rf }} / f_{\text {rf }}$, as shown in Fig. 6, and by fitting Eq. (21) to the measurement data, the obtained values for $\alpha_{1}, \alpha_{2}$, and $\alpha_{3}$ were $5.0 \times 10^{-6}, \quad-1.8 \times 10^{-4}, \quad$ and $1.1 \times 10^{-1}$, respectively.

The discrepancy between the measured and theoretical values could be due to several reasons such as the mea-

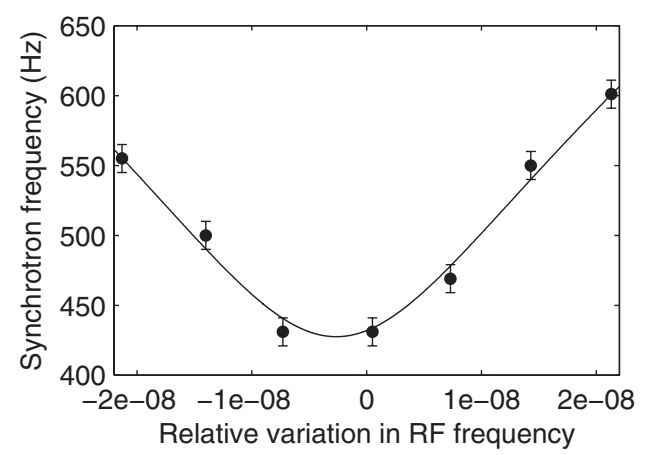

FIG. 6. Measured variation of the synchrotron frequency with respect to the relative variation in the rf frequency. The dots are the measurement data with their error bars of $\pm 10 \mathrm{~Hz}$. The solid curve corresponds to a fit of Eq. (21) in the case of a $\alpha / 100$ low- $\alpha$ optics: $\alpha_{1}=5.0 \times 10^{-6} \pm 4 \times 10^{-7}, \quad \alpha_{2}=-1.8 \times$ $10^{-4} \pm 4 \times 10^{-5}$, and $\alpha_{3}=1.1 \times 10^{-1} \pm 2 \times 10^{-2}$.

surement errors for low values of synchrotron frequency, machine nonlinearities not taken into account in the model, and the high sensitivity of the $\alpha_{2}$ value to the variation of the sextupole strengths for very low- $\alpha$ optics [4].

\section{THE THREE STORED BEAM EVENT SUPPORTED BY SIMULATION}

In this part, the event of the three beams reported in Sec. II and their dynamics in the transverse and longitudinal phase spaces, when the rf frequency and a focusing sextupole family (SF) strength are modified, are explained based on the theoretical framework set in the previous sections. The Hamiltonian of Eq. (16) is used to simulate different cases of the observed dynamics.

Although the different orders of the momentum compaction factor were not measured in the low- $\alpha$ optics in which the three beams were observed, nevertheless this event can be simulated using realistic parameters.

The simulations were based on the $\alpha_{1} / 20$ optics of which the first-order theoretical value is $\alpha_{1}=2.2 \times$ $10^{-5}$. As a first attempt, using the fact that the imaged off-momentum beams (2) and (3) of Fig. 1(a) are equally displaced from the on-momentum beam (1) with a full spatial separation of $x=3 \mathrm{~mm}$ at the dipole exit port, the energy offsets of the off-momentum beams were deduced from the chromatic orbit using the theoretical dispersion function up to the third order. The solutions found are $\delta_{2}=4.6 \%$ and $\delta_{3}=-8.5 \%$, respectively. Since such energy deviations lead to large off-momentum chromatic closed orbits well beyond the machine physical acceptance, the conclusion was that the model was not the good one to fit the observations. Indeed the optics was distorted during the experiment and it was not possible to measure the optical functions due to technical constraints.

As a second attempt, because of the lack of information on the higher-order terms of dispersion function, the energies of the two off-momentum beams were assumed not 
to exceed $\pm 1.5 \%$ in order to be kept inside the transverse dynamic and physical acceptances (based on computation performed using the TRACY code [22]). Starting with these $\delta$ values, and using the theoretical value of $\alpha_{1}$ the reverse problem was considered. By assuming that the orbit was corrected so that $\chi \approx 4.3 \times 10^{-9}$, then by solving Eq. (12) for $\alpha_{2}$ and $\alpha_{3}$, a new lattice model was obtained with $\alpha_{2}=$ $-1.9 \times 10^{-5}$ and $\alpha_{3}=-9.3 \times 10^{-2}$. By plotting Eq. (16) for these parameters, a longitudinal phase space compatible with Fig. 1(a) is obtained [Fig. 7(a)]. In this case the off-momentum bucket layers (2) with $\delta_{2}=-1.5 \%$, and
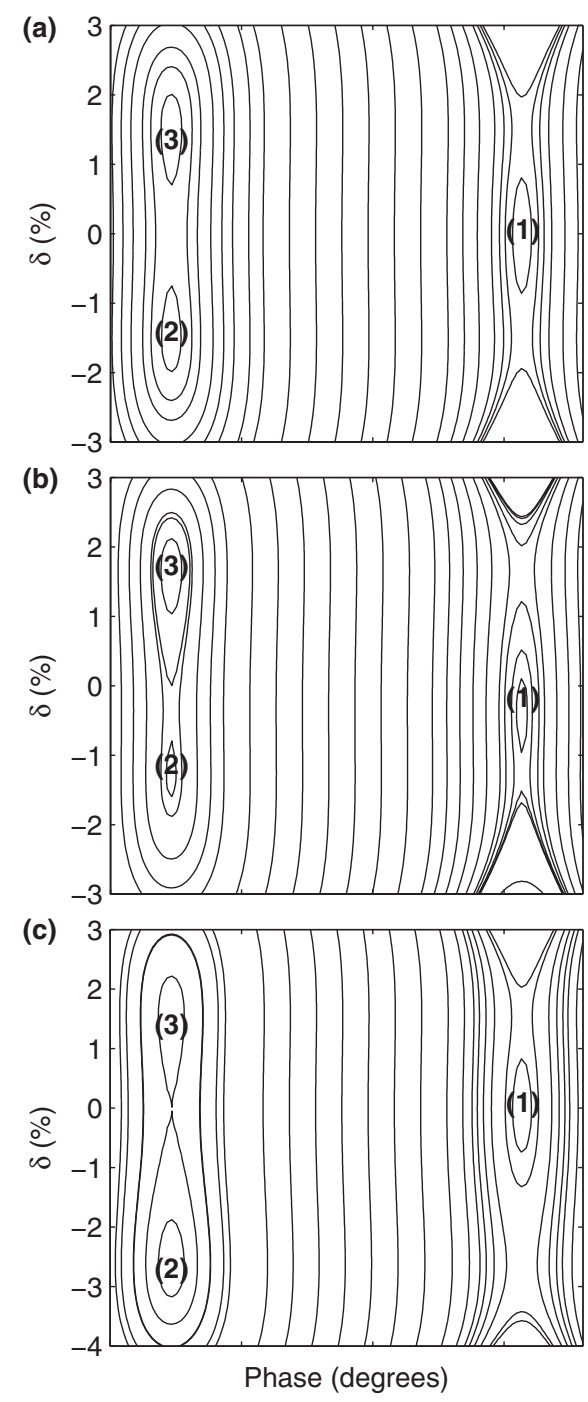

FIG. 7. Longitudinal phase spaces compatible with the threebeam event depicted in Figs. 1(a)-1(c). The two off-momentum beams (2) and (3) and the on-momentum beam (1) are derived from the modified low- $\alpha$ lattice of the SOLEIL storage ring using the parameters $\alpha_{1}=2.2 \times 10^{-5}$ and $\left(\chi, \alpha_{2}, \alpha_{3}\right)$ : $(4.3 \times$ $\left.10^{-9},-1.9 \times 10^{-5},-9.3 \times 10^{-2}\right),\left(7.5 \times 10^{-8},-1.9 \times 10^{-5}\right.$, $\left.-9.3 \times 10^{-2}\right), \quad\left(4.3 \times 10^{-9},-6.0 \times 10^{-4},-5.5 \times 10^{-2}\right)$ respectively for the cases (a), (b), and (c). Case (b) is obtained by increasing the rf frequency by $25 \mathrm{~Hz}$ while case (c) by rising the strength of a SF sextupole by $8 \%$.
(3) with $\delta_{3}=1.5 \%$ are identified to be the additional offmomentum beams while the index (1) denotes the onmomentum beam.

The beam depicted by Fig. 1(b) is the result of a $25 \mathrm{~Hz}$ variation in the rf frequency. Because of the small $\alpha_{1}$ value, even a small variation in rf frequency causes a nonnegligible horizontal orbit distortion that shifts in turn the on-momentum beam energy. The variation in the rf frequency can be more or less represented by the $\chi$ value (see Sec. VI). Hence, varying the rf frequency of $25 \mathrm{~Hz}$ increases for instance the value of $\chi$ by $7.1 \times 10^{-8}$ which shifts the on-momentum beam energy by $-0.36 \%$ towards the beam (2). So using the same above values of the momentum compaction factors and the increased value of $\chi\left(7.53 \times 10^{-8}\right)$, a similar case to Fig. 1(b) can be obtained: its phase space is shown in Fig. 7(b). It should be noted that $\alpha_{2}$ (the most sensitive term to orbit distortion in the low- $\alpha$ optics according to simulations using BETA) was varied by a factor \pm 10 without any significant change in the dynamics shown in Fig. 7(b).

Finally, one considers the case where the SF sextupole family strength is changed while the rf frequency is kept constant. Increasing the strength of the SF sextupole family reduces the $\alpha_{2}$ value which in turn increases the energy gap between on-momentum beam (1) and the negative offmomentum one (2). However, a variation of the sextupole strength modifies the $\alpha_{3}$ value due to its high sensitivity to the nonlinear dispersion. For example, using the $\alpha_{1} / 20$ optics, simulations show that increasing the sextupole strength by $8 \%$ decreases the value of $\alpha_{2}$ by a factor 34 from $-2 \times 10^{-5}$ to $-6.9 \times 10^{-4}$ and increases $\alpha_{3}$ by $43 \%$ from $9.2 \times 10^{-2}$ to $1.35 \times 10^{-1}$. Assuming a negligible closed orbit distortion, a similar case to Fig. 1(c) can be obtained by decreasing the $\alpha_{2}$ value by a factor 30 and increasing the $\alpha_{3}$ value by $40 \%$. Figure 7(c) shows that the beam (2) energy is deviated in energy to $\delta_{2}=-2.6 \%$ which exceeds the machine transverse dynamic acceptance for the low- $\alpha$ optics.

Using a realistic adjusted model of the low- $\alpha$ optics to simulate multibeam dynamics, it has been possible to draw longitudinal phase spaces compatible with the observations performed in the SOLEIL storage ring. More refined simulations could be made by using measured values for $\alpha_{1}, \alpha_{2}$, and $\alpha_{3}$, and using the knowledge of dispersion function for each case previously described.

\section{CONCLUSION}

In this paper, the first observation in November 2007 of three beams simultaneously stored into a circular accelerator has been reported. By including the thirdorder momentum compaction factor into the longitudinal equations of motion, it has been possible to explain theoretically, to some extent, the three-beam configuration observed in a low- $\alpha$ operation mode of the SOLEIL storage ring. 
Moreover, due to its large sensitivity at low- $\alpha$ optics, taking the path lengthening momentum-independent term $\chi$ into account was necessary to simulate the observed three-beam dynamics under variation of $r f$ frequency. By including $\alpha_{3}$ and $\chi$ into the longitudinal beam dynamics, it was possible to extend the formula of synchrotron frequency as a function of the relative rf-frequency change which was used to evaluate experimentally the first three terms of momentum compaction factor for a low- $\alpha$ optics.

The analysis of three typical cases has shown, theoretically and experimentally, the high sensitivity of the longitudinal phase-space behavior to the residual orbit error, the rf frequency, and the sextupole strength, through the value of $\chi$ and of the higher-order terms of momentum compaction factor.

The value of the third-order momentum compaction factor is a key parameter to obtain several stable beams in low- $\alpha$ operation. Then, similar to the operation at the Metrology Light Source, the third-order momentum compaction could be adjusted by inserting octupole magnets into the SOLEIL magnet lattice to better control the longitudinal beam dynamics for extremely low- $\alpha$ operation.

\section{ACKNOWLEDGMENTS}

The authors are grateful to the operation, diagnostics, and power supply groups for their support during these experiments. They thank M.-E. Couprie and M. Labat for the bunch length measurements performed with a picosecond streak camera.

[1] J.-M. Filhol, A. Nadji et al., in Proceedings of the 2008 European Particle Accelerator Conference (EPS-AG, Genoa, Italy, 2008), pp. 2022-2024.

[2] J. Barros, L. Manceron, J.-B. Brubach, G. Creff, C. Evain, M.-E. Couprie, A. Loulergue, L. Nadolski, M.-A. Tordeux, and P. Roy, J. Phys. Conf. Ser. 359, 012002 (2012).

[3] C. Evain et al., in Proceedings of the IEEE 35th International Conference on Infrared Millimeter and Terahertz Waves (IRMMW-THz) (Rome, Italy, 2010), p. 1 .
[4] M. Attal, Ph.D. dissertation, Paris XI University, Paris, 2009.

[5] P. Brunelle et al., in Proceedings of the Second International Particle Accelerator Conference, San Sebastián, Spain (EPS-AG, Spain, 2011), pp. 2124-2126.

[6] M. Tordeux et al., in Proceedings of the Third International Particle Accelerator Conference, New Orleans, Louisiana, 2012 (IEEE, Piscataway, NJ, 2012), pp. 1608-1609.

[7] H. Wiedemann, Particle Accelerator Physics II (SpringerVerlag, Berlin, Heidelberg, 1999), Sect. 6.3.

[8] A. Loulergue, in the XV European Synchrotron Light Source Workshop, Diamond, UK, 2007.

[9] J. B. Murphy and S. L. Kramer, Phys. Rev. Lett. 84, 5516 (2000).

[10] M. Ries, in the XVIII European Synchrotron Light Source Workshop, Trieste, Italy, 2010.

[11] M. Ries et al., in Proceedings of the Second International Particle Accelerator Conference, San Sebastián, Spain (Ref. [5]), pp. 945-947.

[12] M. Ries et al., in the Low Emittance Ring Workshop, Heraklion, Greece, 2011.

[13] The BETA code, J. Payet, CEA/DSM/Irfu/SACM.

[14] A. Riabko, M. Bai, B. Brabson, C. Chu, X. Kang, D. Jeon, S. Lee, and X. Zhao, Phys. Rev. E 54, 815 (1996).

[15] D. Robin, E. Forest, C. Pellegrini, and A. Amiry, Phys. Rev. E 48, 2149 (1993).

[16] J. Le Duff, CERN Accelerator School Report No. CERN 94-01, 1992, Vol. 1, pp. 30-52.

[17] W. Pirkl, CERN Accelerator School Report No. CERN 9506, 1993, Vol. 1, pp. 233-258.

[18] H. Goldstein, Classical Mechanics (Addison-Wesley, Reading, MA, 1980), 2nd ed.

[19] Handbook of Mathematical Functions with Formulas, Graphs, and Mathematical Tables, edited by $\mathrm{M}$. Abramowitz and I. A. Stegun (Dover Publications, New York, 1972), p. 17.

[20] K-Y. Ng, Nucl. Instrum. Methods Phys. Res., Sect. A 404, 199 (1998).

[21] A. Nadji, P. Brunelle, G. Flynn, M. P. Level, M. Sommer, and H. Zyngier, Nucl. Instrum. Methods Phys. Res., Sect. A 378, 376 (1996).

[22] H. Nishimura, J. Bengtsson, and E. Forest, TRACY2 User's Manual (unpublished). The SOLEIL version of TRACY3 is used for performing the simulation. 